\title{
Programas de optimización del uso de antimicrobianos en Perú: Un acuerdo sobre lo fundamental
}

\author{
Cristhian Hernández-Gómez', Luis Hercilla², Fernando Mendo³, Giancarlo Pérez-Lazo4, \\ Elías Contreras ${ }^{3}$, Eresvita Ramírez², Wilfredo Flores', Ángela Julca², Nancy Chuquiray², Berenice Arenas ${ }^{4}$ \\ Susan Abarca4, Mario Viñas 3 , Elba Linares³, María Virginia Villegas' y Luis Ricardo Illescas4.
}

\section{Antimicrobial Stewardship programs in Peru: A fundamental agreement}

Background. Antimicrobial resistance (AMR) is a global threat to public health. Antibiotic stewardship programs (AMSP) promote the proper use of antimicrobials, improve clinical and economic outcomes, and helps containing the AMR. Aim: To evaluate the diagnostic phase of the AMS programs and early implementation of AMS at three high complexity hospitals that belong to the social security system in Peru. Methods: A quasi-experimental multicenter study was implemented. The construction of the AMSP, microbiological baselines, antimicrobial consumption and consensus on AMS activities were evaluated at the diagnosis and early implementation periods of the AMSP. Results: Following implementation, hospitals doubled their score of resources and processes available for the AMS program from 6.75 to 13.75. The prevalence of extended spectrum beta-lactamase producing enterobacteria was 50-60\% while Pseudomonas aeruginosa averaged 69\% resistance to carbapenems. The defined daily dose (DDD) of ceftriaxone was 13.63, vancomycin 7.35 and meropenem 6.73 in average. Hospitals A and C decreased the use of antimicrobials (30-50\%). Discussion: The implementation of the AMSP in the three hospitals was achieved through diverse strategies designed by multidisciplinary teams, which in addition to its articulation, reduce the consumption of broad spectrum antimicrobials at an early stage.

Keywords: Antimicrobial stewardship; drug resistance; multiple; consensus.

Palabras clave: Programas de optimización del uso de los antimicrobianos; fármaco-resistencia microbiana; consenso.

\section{Introducción}

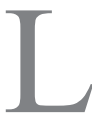

a resistencia a los antimicrobianos (RAM) es una amenaza para la Salud Pública mundial. El surgimiento de la RAM es multifactorial, espontánea, adquirida mediante transmisión de material genético móvil entre bacterias y puede seleccionarse por la exposición a los antimicrobianos de uso en la medicina clínica, medicina veterinaria y de aplicación industrial ${ }^{1,2}$. La Organización Mundial de la Salud, en su primer informe de vigilancia mundial de la resistencia a los antimicrobianos (2014), reportó el incremento de cinco bacterias multi-drogo-resistentes (MDR) a nivel global, tales como Escherichia coli resistente a cefalosporinas de tercera generación y quinolonas, Klebsiella pneumoniae resistente a cefalosporinas de tercera generación y carbapenémicos además de Staphylococcus aureus resistente a meticilina en ambientes hospitalarios ${ }^{3}$. La RAM no se limita a infecciones bacterianas e involucra también las infecciones fúngicas, las que generan presentaciones clínicas invasoras con ya limitadas opciones terapéuticas ${ }^{4}$.
La RAM amenaza la práctica de procedimientos quirúrgicos básicos, así como los más avanzados procedimientos en medicina, ya que actualmente hay alta proporción de infecciones asociadas a la atención en salud (IAAS) causadas por estos microorganismos MDR 5 . Los problemas asociados a la RAM trascienden a la salud individual de los pacientes, los profesionales y las organizaciones de salud ${ }^{6}$. Se estima que 700.000 personas mueren anualmente debido a infecciones por organismos MDR y que para el año 2050, esta cifra puede ascender a los 10 millones de vidas ${ }^{7}$. El Banco Mundial proyecta que, para ese mismo año, el producto interno global podría disminuir entre 1,1 y $3,8 \%$ anualmente debido al fenómeno de la RAM ${ }^{8}$.

Las causas de la RAM son un problema multifactorial; sin embargo, existe una alta correlación entre el uso indiscriminado de los antimicrobianos y la emergencia de microorganismos $\mathrm{MDR}^{9}$. Para países en vías de desarrollo, los niveles de resistencia pueden explicarse, adicionalmente, por las prácticas de automedicación, compra de antimicrobianos de venta libre, ausencia de políticas que promuevan el uso racional de los antimicrobianos,
'Grupo de investigaciones en Resistencia Antimicrobiana y Epidemiología Hospitalaria (RAEH), Universidad El Bosque, Bogotá D.C., Colombia. ${ }^{2}$ Hospital Nacional Alberto Sabogal Sologuren, EsSalud. Lima, Perú.

${ }^{3}$ Hospital Nacional Edgardo Rebagliati Martins, EsSalud. Lima, Perú.

${ }^{4}$ Hospital Nacional Guillermo Almenara Irigoyen, EsSalud. Lima, Perú.

Conflictos de interés. Cristhian Hernández-Gómez y María Virginia Villegas han recibido honorarios por consultoría, conferencias científicas o apoyo a la investigación de Merck Sharp \& Dohme y Pfizer. Los otros autores declaran no tener conflictos de interés para este artículo.

El presente estudio no tuvo fuentes de financiación o patrocinios. Fue realizado con recursos propios de las instituciones participantes.

Recibido: 2 de diciembre de 2018 Aceptado: 26 de agosto de 2019

Correspondencia a: Giancarlo Pérez-Lazo giancarlo.perez@unmsm.edu.pe 
sumado a las limitaciones en recursos para la prevención y el control efectivo de las IAAS ${ }^{10}$. De igual manera, la ausencia de recursos diagnósticos oportunos y precisos se consolida cada vez más como el "talón de Aquiles" de la contención de la resistencia a los antimicrobianos ${ }^{11}$.

Con el propósito de reducir la presión selectiva que favorece la aparición de microorganismos MDR, se han implementado diferentes estrategias como los programas de optimización o gerenciamiento del uso de antimicrobianos (PROA). Los PROA son programas coordinados cuyo objetivo principal es promover el uso adecuado de los antimicrobianos, mejorar los resultados clínicos y económicos de la atención en salud de los pacientes, contener la emergencia de la RAM y contribuir a la disminución de la diseminación de infecciones causadas por microorganismos MDR de la mano de los programas de prevención y control de infecciones ${ }^{12}$.

Para la implementación de los PROA, son necesarios equipos multidisciplinarios con capacidad de liderazgo en las instituciones para orientar la optimización de las terapias antimicrobianas, además de promover la adecuada selección, dosificación, ruta de administración y duración de los mismos ${ }^{13}$. Con la finalidad de cumplir sus objetivos, los equipos de los PROA implementan estrategias como la auditoría prospectiva, la restricción en el formulario, la prescripción pre-autorizada, algoritmos o flujogramas de terapia empírica para la prescripción o modificación de la terapia, uso de programas informáticos que apoyan la decisión terapéutica y la combinación de tecnologías que favorecen el diagnóstico rápido en los laboratorios de microbiología ${ }^{14}$.

El objetivo de este estudio fue evaluar las fases de diagnóstico e implementación temprana de los programas de optimización del uso de antimicrobianos en tres instituciones hospitalarias de alta complejidad en Perú.

\section{Materiales y Métodos}

Se condujo un estudio ambispectivo, multicéntrico, cuasi experimental, en tres hospitales de alta complejidad en Lima, Perú. Las variables fueron evaluadas en tres períodos, para el diagnóstico, 18 meses antes de la implementación (2016-Semestre I de 2017), estructuración del programa (Semestre II de 2017) y tres meses de fase temprana de implementación del programa (enero-marzo de 2018). Los tres hospitales participantes (A, B y C) fueron instituciones médico-quirúrgicas pertenecientes al Sistema de Seguridad Social del Perú (EsSalud), con servicios de onco-hematología, trasplantes de órganos sólidos y unidades de cuidado intensivo neonatal, pediátrico y adultos. La institución A cuenta con 1.100 camas, la institución $\mathrm{B}$ con 1.744 camas mientras que la $\mathrm{C}$ cuenta con 500 camas, incluyendo las camas habilitadas para hospitalización en servicios de emergencia.

\section{Diagnóstico y seguimiento de recursos para la implementación de PROAs}

Para el diagnóstico de procesos y recursos disponibles para la implementación de los programas, se aplicó el índice modificado de programas de optimización del uso de antimicrobianos $(\mathrm{ICATB}=I C A T B=$ Indice Composite de bon usage des AnTiBiotiques). Este índice permite medir las características administrativas y asistenciales de la institución, los recursos informáticos disponibles y las actividades enfocadas a la contención del uso indiscriminado de los antimicrobianos, la vigilancia epidemiológica y la evaluación de la prescripción ${ }^{15}$. La aplicación del índice se realizó a través de entrevistas presenciales con los equipos de trabajo, sumado a la verificación de los procesos por vía documental o mediante visita a los servicios de interés. Con posterioridad al diagnóstico, se establecieron ejes de priorización de actividades para la implementación del PROA durante su período de estructuración. La metodología usada fue la de grupo focal que incluyó profesionales de los servicios de infectología, microbiología, inteligencia sanitaria, unidades de cuidado intensivo y servicio farmacéutico ${ }^{16}$. A partir de los ejes priorizados para la implementación del PROA, se analizó el contenido de las discusiones por categorías o áreas de enfoque en cada hospital. Las áreas de enfoque correspondieron a las oportunidades de mejora del servicio de microbiología, servicio farmacéutico, servicio de infectología, unidades de cuidado intensivo y programa de prevención y control de infecciones (Inteligencia Sanitaria). Para cada área de enfoque se construyeron líneas de acción priorizadas. Como medida de seguimiento, seis meses después se aplicó de nuevo el ICATB, siguiendo la metodología anteriormente descrita para el índice.

\section{Estudio de los patrones de la resistencia antimicrobiana}

Para determinar los perfiles epidemiológicos de la resistencia antimicrobiana se usó la base de datos de microbiología del conjunto de las instituciones participantes. Se incluyeron aislados clínicos de pacientes, excluyendo aislados de control de calidad interno, externo o estudios de rastreo microbiológico. Para el período 2016-2017, se realizó el análisis usando el software WHONET ${ }^{\circledR}$ (versión 5.6). La interpretación de los perfiles de susceptibilidad se ajustó según los puntos de corte del Clinical \& Laboratory Standards Institute (CLSI) en sus versiones vigentes al momento del estudio de cada aislado.

\section{Estudio de los patrones de consumo de los antimicrobianos}

Para generar el diagnóstico de las tendencias de consumo de antimicrobianos y evaluar el comportamiento durante la fase temprana de implementación del programa, se tuvieron en cuenta los períodos, 2016-semestre 
I de 2017 y enero-marzo de 2018, respectivamente. El consumo se evaluó con base en la fórmula de dosis diaria definida (DDD) de la Organización Mundial de la Salud (OMS) ${ }^{17}$. Esta fórmula permite ajustar el consumo de antimicrobianos del conjunto de las instituciones, de acuerdo con la ocupación hospitalaria y el número total de camas. Se calcularon las DDD globales para el conjunto de las instituciones de los siguientes antimicrobianos intravenosos: ceftriaxona, piperacilina/tazobactam, imipenem, meropenem, ertapenem, colistina, ciprofloxacina, vancomicina y caspofungina.

\section{Consenso para el abordaje empírico de patologías infecciosas priorizadas}

Usando la metodología de grupo focal ${ }^{16}$, durante la fase de estructuración de los PROAs y a través de reuniones con los equipos multidisciplinarios de cada hospital, se evaluaron las alternativas terapéuticas de las patologías infecciosas prevalentes en función del comportamiento microbiológico y de los patrones de consumo de antimicrobianos de cada institución.

\section{Consenso para la prevención, contención y manejo de infecciones por microorganismos MDR}

De igual manera, junto con los equipos de los PROA y con base en las tendencias de los microorganismos MDR de mayor impacto para las instituciones, se identificaron las mejores estrategias para la prevención, contención y abordaje terapéutico para infecciones por bacterias MDR, según la prevalencia de los períodos 2016-2017 de cada institución.

\section{Resultados}

Al aplicar el ICATB durante la fase de diagnóstico, las tres instituciones tuvieron una puntuación global promedio de 6,75 , ubicándose en el percentil 30 de la categoría de clínicas u hospitales de $\geq 300$ camas (Tabla 1). Los tres hospitales contaban con equipos de infectología que ejercían la restricción de uso de antimicrobianos.

Los criterios que tuvieron calificación 0 , fueron la ausencia de equipos multidisciplinarios dedicados a la ejecución de actividades del programa, flujogramas o algoritmos de terapia empírica antimicrobiana, la vigilancia del consumo de antimicrobianos o la no evaluación de los patrones de prescripción, entre otros (Tabla 1). A posteriori del diagnóstico de recursos y procesos, los equipos de trabajo de las tres instituciones lograron consenso sobre las áreas de enfoque del servicio farmacéutico, infectología y unidades de cuidado intensivo. En contraste, los servicios de microbiología e inteligencia sanitaria no llegaron a consenso en las líneas de acción de diagnóstico oportuno y de las estrategias de vigilancia mensual de microorganismos MDR, continuando cada una con su propia estrategia (Tabla 2).

El diagnóstico de las tendencias del comportamiento microbiológico estableció que, en promedio para los tres hospitales, E. coli (>40\%) y K. pneumoniae (10\%) son las bacterias predominantes en los servicios de hospitalización general y servicios de emergencias, mientras que las unidades de cuidado intensivo presentan 19\% de aislamiento de Staphylococcus epidermidis como bacteria más frecuente, seguido de Pseudomonas aeruginosa (14\%) y Acinetobacter baumannii (10\%) (Figura 1). El análisis de los fenotipos con resistencia a cefalosporinas de tercera generación sugiere la presencia de $\beta$-lactamasas de espectro extendido (BLEE's) en $E$. coli (54\%) y $K$. pneumoniae $(64 \%)$ en servicios de hospitalización y emergencias, donde, además, $49 \%$ en promedio de los aislados de $S$. aureus son resistentes a oxacilina. Por otro lado, en unidades de cuidado intensivo, la resistencia supera el $75 \%$ de los aislados para ambas bacterias en todas las instituciones. Además, fue notoria la resistencia de E. coli a ciprofloxacina superando el $67 \%$ de resistencia en las tres áreas de localización. En UCI, P. aeruginosa promedia $69 \%$ de resistencia a carbapenémicos tipo imipenem y meropenem, proporción que es de $42 \%$ promedio en servicios de emergencias (Tabla 3 ).

El diagnóstico del consumo de antimicrobianos demostró que, en promedio para los tres hospitales, los cuatro antimicrobianos de mayor consumo son ceftriaxona (13,63 DDD), vancomicina (7,35 DDD), meropenem (6,73 DDD) e imipenem con 4,76 DDD x 100 días-cama. Para el período comprendido entre agosto de 2016 y marzo de 2017, se aumentó el consumo de meropenem en $82 \%$ mientras que el incremento para imipenem fue de $18 \%$. A junio de 2017, el consumo de piperacilina/tazobactam había disminuido en 55\% (6,07 DDD vs 2,68 DDD) a partir del mes agosto de 2016 (Figura 2).

Posteriormente a la fase de estructuración de los programas y durante el seguimiento en la fase de implementación temprana del PROA, los hospitales A y C tuvieron una puntuación global de 13,75 (percentil $<70$ ), debido a la estructuración de los equipos PROA, incorporación de las estrategias de vigilancia del consumo de antimicrobianos y de la evaluación de la prescripción. El hospital A designó a un médico encargado del PROA con respaldo de la gerencia del hospital, y a un grupo de médicos infectólogos para visitas hospitalarias conjuntas en los servicios quirúrgicos, hematología-trasplante y emergencia. El hospital C se enfocó en reuniones con los diversos servicios clínicos y quirúrgicos para la difusión del mapa microbiológico y supervisar el funcionamiento de los flujogramas de manejo antimicrobiano empírico en conjunto con farmacia, con posterior retroalimentación a las jefaturas de los servicios y la gerencia del hospital.

El hospital B tuvo una puntuación de 11,25, donde 


\begin{tabular}{|c|c|c|c|c|c|c|c|}
\hline \multirow[t]{2}{*}{ Criterio } & \multirow[t]{2}{*}{ Puntuación } & \multicolumn{2}{|c|}{ Hospital A } & \multicolumn{2}{|c|}{ Hospital B } & \multicolumn{2}{|c|}{ Hospital C } \\
\hline & & $\mathrm{M} 0$ ** & M6 $6^{* * *}$ & Mo & M6 & Mo & M6 \\
\hline Nivel de dedicación del equipo PROA* & $\begin{array}{l}1 \text { reunión mensual }=1 \text { punto } \\
2 \text { reuniones mensuales }=2 \text { puntos } \\
3 \text { reuniones mensuales }=3 \text { puntos } \\
>3 \text { reuniones mensuales }=4 \text { puntos }\end{array}$ & 0 & 1 & 0 & 1 & 0 & 1 \\
\hline $\begin{array}{l}\text { Existencia de un referente en la prescripción } \\
\text { de antimicrobianos }\end{array}$ & $\begin{array}{l}\text { Ausente }=0 \text { puntos } \\
\text { Presente }=4 \text { puntos }\end{array}$ & 4 & 4 & 4 & 4 & 4 & 4 \\
\hline Registros clínicos digitales & $\begin{array}{l}\text { Ausente }=0 \text { puntos } \\
\text { Presente }=1 \text { puntos }\end{array}$ & 1 & 1 & 1 & 1 & 1 & 1 \\
\hline Prescripción de antimicrobianos computarizada & $\begin{array}{l}\text { Ausente }=0 \text { puntos } \\
\text { Parcial }=1 \text { puntos } \\
\text { Presente }=2 \text { puntos }\end{array}$ & 1 & 1 & 1 & 1 & 1 & 1 \\
\hline Entrenamiento a quienes prescriben los antimicrobianos & $\begin{array}{l}\text { Ausente }=0 \text { puntos } \\
\text { Presente }=1 \text { puntos }\end{array}$ & 0 & 0 & 0 & 0 & 0 & 0 \\
\hline Guías de uso de antimicrobianos & $\begin{array}{l}\text { Ausente }=0 \text { puntos } \\
\text { Parcial }=1 \text { puntos } \\
\text { Presente }=2 \text { puntos }\end{array}$ & 0 & 1 & 0 & 1 & 0 & 1 \\
\hline Lista de antimicrobianos disponibles para la prescripción & $\begin{array}{l}\text { Ausente }=0 \text { puntos } \\
\text { Presente }=0,25 \text { puntos }\end{array}$ & 0,25 & 0,25 & 0,25 & 0,25 & 0,25 & 0,25 \\
\hline Lista de antimicrobianos con restricción de despacho & $\begin{array}{l}\text { Ausente }=0 \text { puntos } \\
\text { Presente }=0,5 \text { puntos }\end{array}$ & 0,5 & 0,5 & 0,5 & 0,5 & 0,5 & 0,5 \\
\hline $\begin{array}{l}\text { Control de los tiempos de administración } \\
\text { de antimicrobianos durante la terapia }\end{array}$ & $\begin{array}{l}\text { Ausente }=0 \text { puntos } \\
\text { Presente }=0,25 \text { puntos }\end{array}$ & 0 & 0 & 0 & 0 & 0 & 0 \\
\hline Vigilancia del consumo de antimicrobianos & $\begin{array}{l}\text { Ausente }=0 \text { puntos } \\
\text { Presente }=2,5 \text { puntos }\end{array}$ & 0 & 2,5 & 0 & 2,5 & 0 & 2,5 \\
\hline Evaluación de la prescripción de antimicrobianos & $\begin{array}{l}\text { Ausente }=0 \text { puntos } \\
\text { Presente }=2,5 \text { puntos }\end{array}$ & 0 & 2,5 & 0 & 0 & 0 & 2,5 \\
\hline Puntaje global & & 6,75 & 13,75 & 6,75 & 11,25 & 6,75 & 13,75 \\
\hline
\end{tabular}

se destaca la ausencia de estrategias de evaluación de la prescripción y retroalimentación (Tabla 1) en suma a los problemas administrativos como el cambio de gerencia. Durante estas fases, las tres instituciones lograron consenso para la construcción de algoritmos de manejo terapéutico de las infecciones prevalentes en el marco del comportamiento microbiano local y del consumo de antimicrobianos. De igual manera, se logró consenso sobre el control del despacho o regulación del consumo de antimicrobianos de amplio espectro, además de proponer la incorporación de nuevos antimicrobianos al formulario institucional como fosfomicina IV, polimixina $\mathrm{B}$, ceftazi- dima/avibactam y ceftolozano/tazobactam para el manejo de infecciones por microorganismos MDR (Tabla 4).

La tendencia del consumo de antimicrobianos durante la fase de implementación temprana del PROA (eneromarzo de 2018) demostró una disminución en las DDD de meropenem, imipenem, ertapenem, piperacilina/ tazobactam, ceftriaxona y vancomicina en el hospital C (Figura 3). El hospital A presentó disminución del consumo de imipenem y vancomicina, los demás antimicrobianos no presentaron cambios. En contraste el hospital B no presentó cambios en las tendencias de consumo de antimicrobianos. 
Tabla 2. Líneas de acción priorizadas a partir de las áreas de enfoque que los grupos focales de cada institución hospitalaria seleccionaron durante las sesiones de discusión

Grupo Conformación de equipos multidisciplinarios con representantes de las áreas de diagnóstico, administración de medicamentos, procesos de atención clínica focal de pacientes y servicios de prevención y control de infecciones.

\begin{tabular}{|c|c|c|c|c|c|}
\hline $\begin{array}{l}\text { Áreas de } \\
\text { enfoque }\end{array}$ & Servicio de microbiología & Servicio farmacéutico & Servicio de Infectología & Inteligencia Sanitaria & $\begin{array}{l}\text { Unidad de cuidados inten- } \\
\text { sivos }\end{array}$ \\
\hline
\end{tabular}

Líneas de > Diagnóstico oportuno: > Dosis unitaria de los ATM: acción Reducción del tiempo de toma de muestra y notificación de resultados $(2 / 3)^{*}$

> Epidemiología de la microbiología:

Estandarización del sistema de información y uso de software WHONET para análisis de la información. (3/3)

$>$ Estudio de mecanismos de RAM:

Implementación de pruebas de tamizaje para detección de carbapenemasas en enterobacterias, estudio de BLEEs y EVR** (3/3) Consolidación de la estrategia y ampliación a servicios de emergencias (3/3)

> Epidemiología del consumo de los ATM:

Estandarización del sistema de la información y uso de metodología DDDOMS $^{* * *}$. (3/3)

Regulación del despacho: Despacho controlado en las primeras $48-72 \mathrm{~h}$, corte de tratamiento sin interconsulta o seguimiento por infectología (3/3) cia a las pautas:
Algoritmos de terapia empírica:

Algoritmos por patologías infecciosas más prevalentes $(3 / 3)$

Vigilancia de organismos MDR: Seguimiento mensual del índice de organismos $\mathrm{MDR}^{\wedge}$ y vigilancia activa de brotes (2/3)

Verificación de los 4 correctos del PROAt:

Modificación de vía, dosis o duración y corte de tratamientos sin indicación $(3 / 3)$

$>$ Listas de chequeo para la prevención de las IAASf: Priorización de vigilancia a dispositivos invasivos (CVC, CU y VM) (3/3)

$>$ Verificación de la adherenConstrucción de indicadores de adherencia a algoritmos o des-escalamiento $(3 / 3)$
Estrategias de manejo terapéutico de infecciones por organismos MDR: Estandarización de tratamientos para el manejo de infecciones por organismos MDR, estrategias de des-escalamiento para estas infecciones y medidas de contención en el marco de las políticas de control de infecciones (3/3)

${ }^{*}(X / X)=$ Número de hospitales que lo definieron como línea de acción / número de hospitales participantes. BLEE's y ERV= $\beta$-lactamasas de espectro extendido y Enterococcus spp. resistente a vancomicina. DDD = Dosis Diaria Definida - Organización Mundial de la Salud. +PROA = Programa de optimización del uso de antimicrobianos. ${ }^{\wedge} \mathrm{MDR}=$ Multi-drogo-resistencia. fIAAS = Infecciones Asociadas a la Atención en Salud. $\$$ CVC, CU y VM = catéter venoso central, catéter urinario y ventilación mecánica.

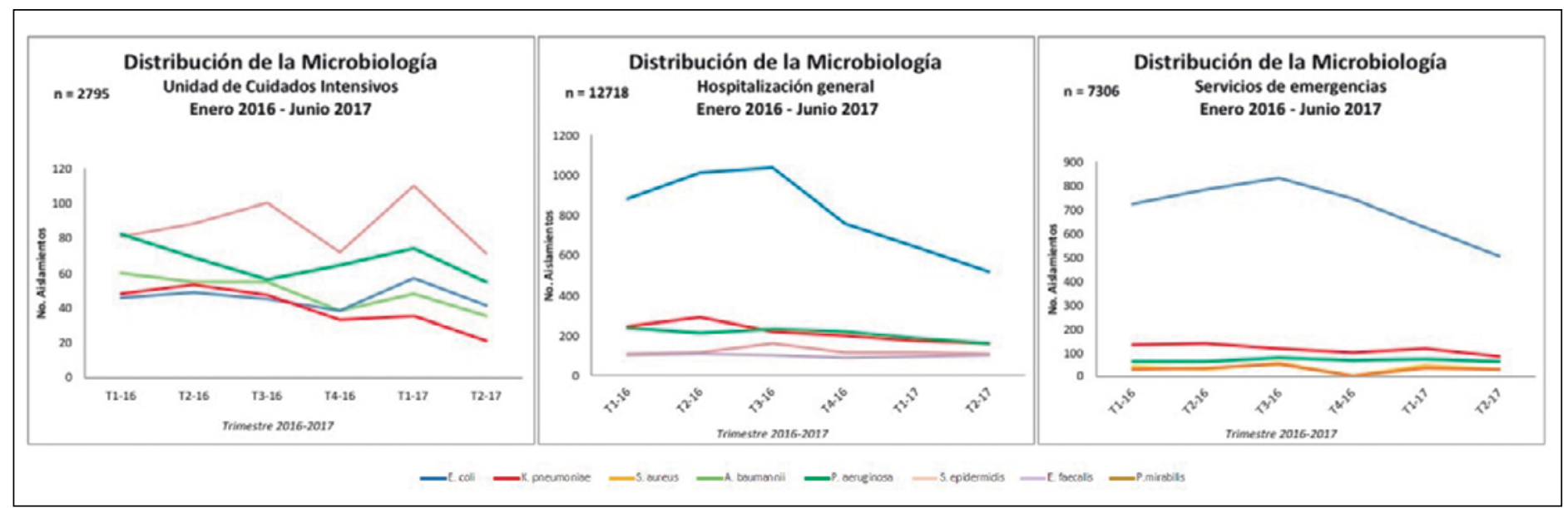

Figura 1. Distribución y tendencia de las bacterias más frecuentes por tipo de localización. Período de estudio: enero de 2016 a junio de 2017. 


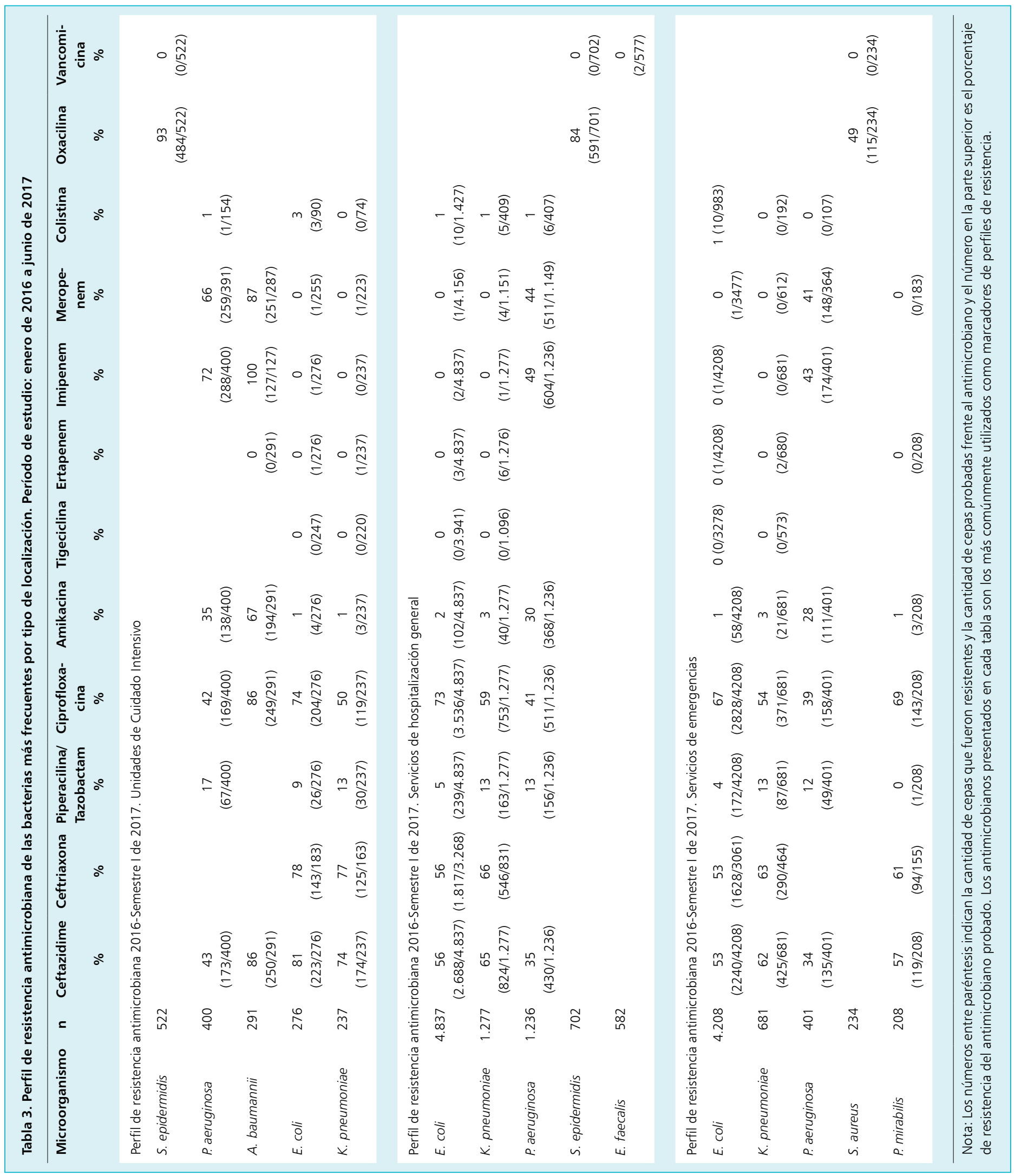




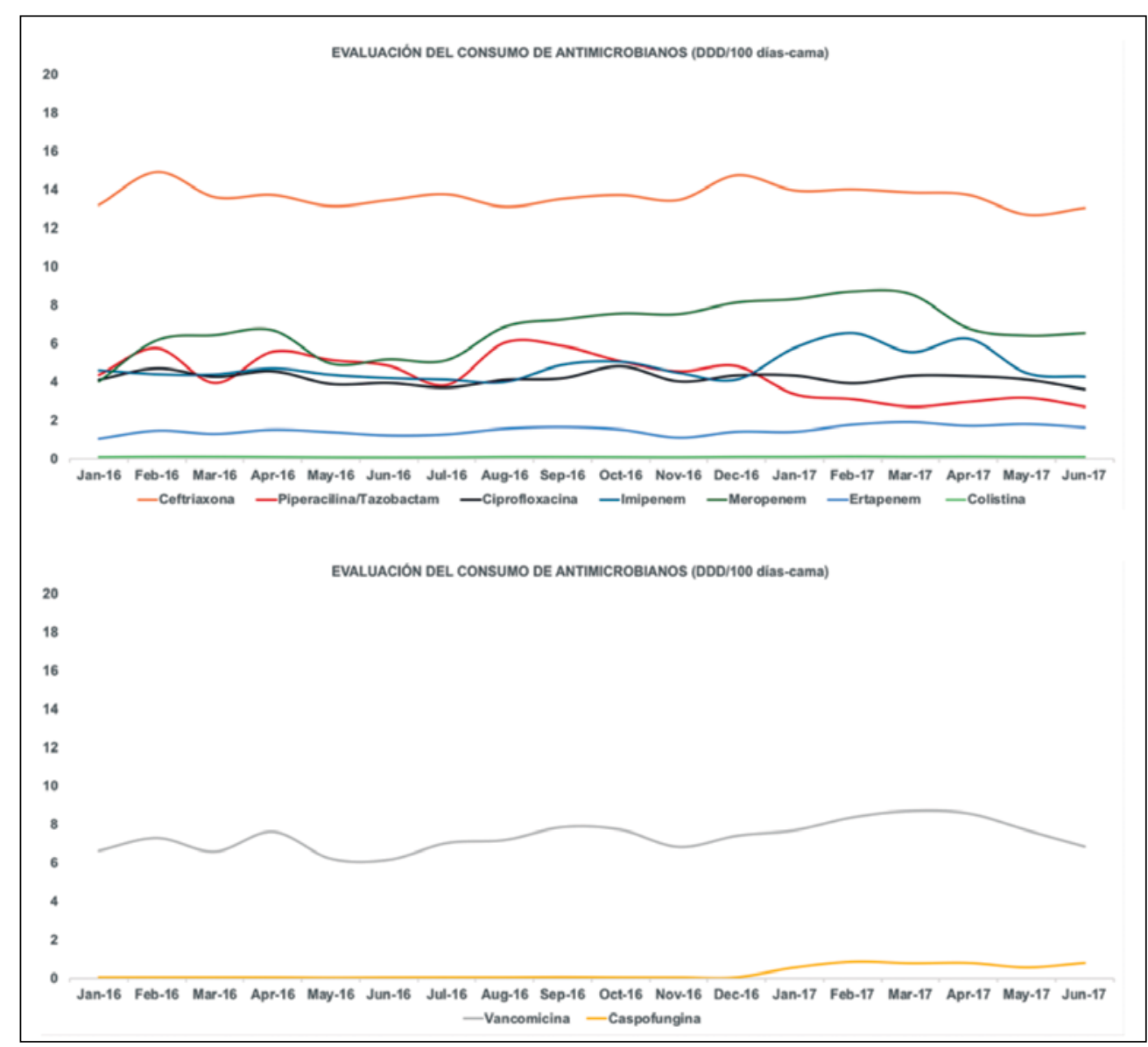

Figura 2. Fase de diagnóstico del consumo de antimicrobianos. Tendencia del consumo de antimicrobianos de amplio espectro en dosis diaria definida (DDD/100 días-cama). Período de estudio: enero de 2016 a junio de 2017.

Tabla 4. Líneas de acción priorizadas a partir de las áreas de enfoque para el abordaje terapéutico empírico de patologías infecciosas priorizadas y manejo de infecciones por microorganismo MDR

Grupo focal Consenso para el abordaje terapéutico empírico de patologías infecciosas priorizadas y manejo de infecciones por microorganismos MDR $^{\wedge}$

Áreas de Patologías infecciosas prioenfoque rizadas

Líneas de > Según prevalencia local: acción Infecciones de tracto urinario, infecciones de piel y tejidos blandos (incluyendo pie diabético), infección intra abdominal y neumonía hospitalaria $(3 / 3)^{*}$

$>$ Algoritmos de manejo: De acuerdo a la epidemiología local de la microbiología y del consumo de antimicrobianos, se construyeron algoritmos de manejo empírico (3/3)
Antimicrobianos con regulación de despacho

> Según epidemiología local: Estrategias de control del consumo de ceftriaxona, ciprofloxacina, vancomicina y regulación del consumo de imipenem y meropenem (3/3)

$>$ Incorporación de antimicrobianos a formulario institucional: Incorporación de ertapenem, tigeciclina y fosfomicina trometamol (3/3)
Estratificación de la infección por factores de riesgo

> Estratificación de la infección: Considerar factores de riesgo epidemiológicos y escala de gravedad de las infecciones en la elección del tratamiento antimicrobiano (3/3)
Vigilancia activa de infecciones por organismos MDR

$>$ Rastreo de microorganismos MDR: Fomentar el rastreo de enterobacterias carbapenem resistente, enterococo vancomicina resistente y Acinetobacter baumannii MDR (2/3).

$>$ Verificar medidas de aislamiento de contacto: Vigilar y verificar el cumplimiento de las medidas de aislamiento de contacto en los servicios de atención (2/3)
Abordaje terapéutico de las infecciones por microorganismos MDR

> Terapia combinada en infecciones por microorganismos MDR: Uso de terapia combinada en infecciones por MDR con seguimiento de respuesta microbiológica para posterior ajuste de terapia (3/3)

- Incorporación de antimicrobianos a formulario institucional: Ampliación de cobertura terapéutica o perfil de seguridad en el tratamiento con antimicrobianos tipo fosfomicina IV**, polimixina $B$, ceftazidime/avibactam y ceftolozane/tazobactam, meropenem/vaborbactam (3/3)

${ }^{*}(X / X)=$ Número de hospitales que lo definieron como línea de acción / número de hospitales participantes. ${ }^{\wedge} \mathrm{MDR}=$ Multi-drogo-resistencia. ${ }^{* *} \mathrm{IV}=$ intravenoso. 
Figura 3. Fase de implementación temprana del PROA en hospitales $A$ y $C$. Tendencia del consumo de antimicrobianos de amplio espectro en dosis diaria definida (DDD/100 díascama). Período de estudio: enero-marzo de 2018.

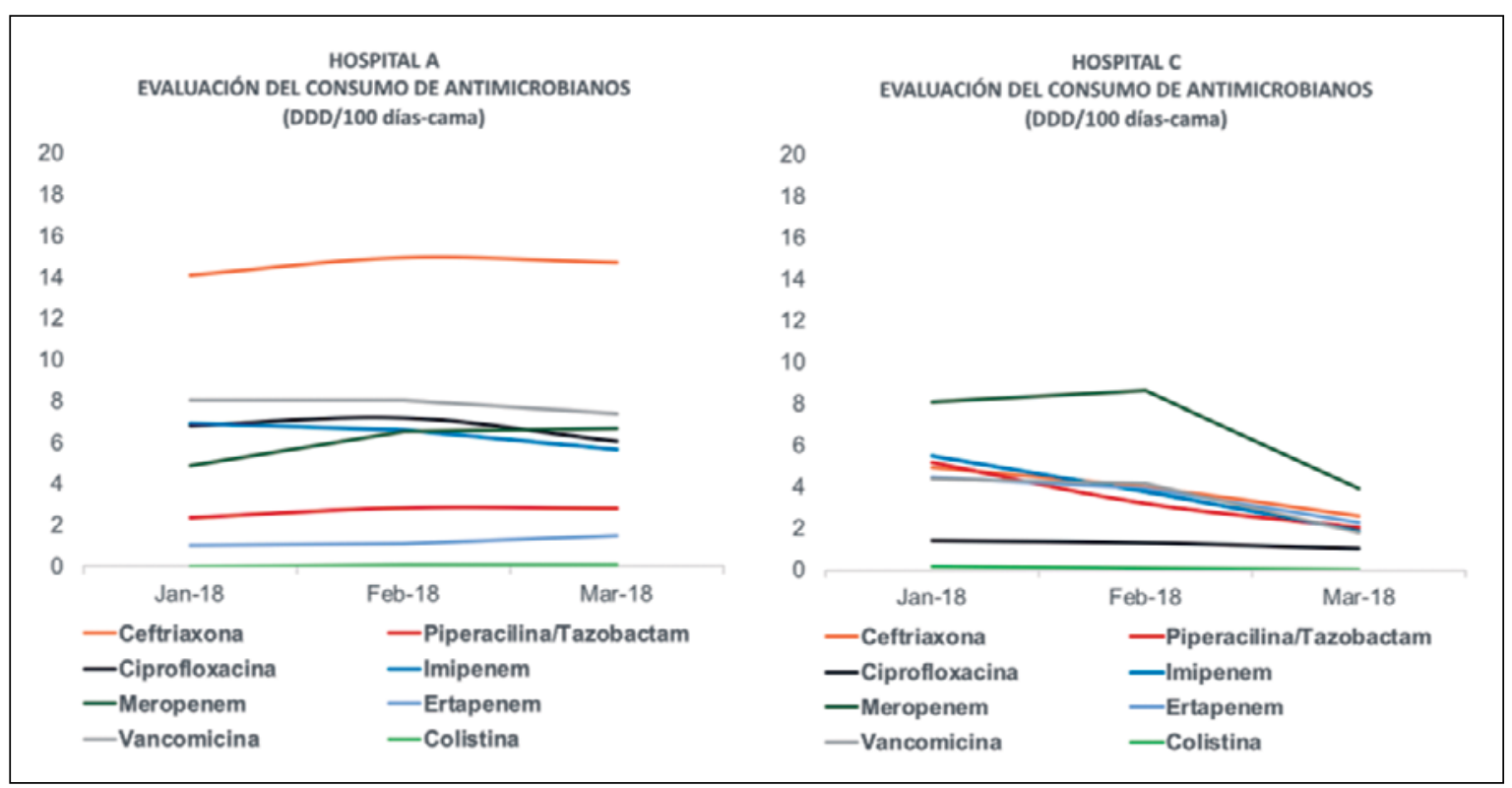

\section{Discusión}

Aunque los programas de optimización de antimicrobianos se han estado implementando a lo largo del mundo, no existe un consenso claro sobre el conjunto de intervenciones específicas que deben realizar este tipo de programas para garantizar la efectividad de sus estrate$\operatorname{gias}^{18}$. La designación de un líder que dirija el programa y la aproximación multidisciplinaria en el abordaje de las problemáticas a las que se enfrentan los programas, es uno de los mayores retos que se debe considerar en la implementación de los PROA. Lo anterior deberá sumarse a las actividades de intervención y seguimiento de los indicadores que se hayan priorizado al momento de establecer la estrategia ${ }^{19}$. El significativo incremento en la puntuación del índice ICATB entre las fases de diagnóstico y la de implementación temprana del programa $(6,75$ vs 13,75$)$ demuestran que la consolidación de los equipos de trabajo, la vigilancia del consumo de los antimicrobianos, del comportamiento epidemiológico de la RAM y la evaluación de la prescripción pudieron ser elementos fundamentales en la construcción del programa, todos elementos importantes en la fase inicial de la implementación de PROAs como se ha reportado en hospitales canadienses según Leung y cols. ${ }^{20}$. Aunque los PROAs presentan dificultades para su adecuada implementación, por la escasa participación de los grupos clínicos, la falta de estrategias de cooperación entre los actores involucrados y la falta de apoyo en muchos casos de los entes rectores de las instituciones ${ }^{21}$, los hospitales en este estudio han priorizado el trabajo colaborativo entre el servicio de infectología, servicio farmacéutico, microbiología, control de infecciones y líderes de servicios de cuidados intensivos como uno de los ejes para la consolidación de los programas ${ }^{22}$.

La generación de información local a través de la vigilancia epidemiológica de la RAM y los patrones de consumo de antimicrobianos son los componentes claves para orientar las estrategias de restricción, preautorización, o incluso de los algoritmos o flujogramas de terapia empírica, de acuerdo con el programa nacional de promoción del uso adecuado de antimicrobianos del Reino Unido ${ }^{23}$. Sin embargo, en el contexto latinoamericano, el diagnóstico microbiológico de las infecciones no siempre cuenta con la mejor evidencia mientras que los datos representativos sobre la prevalencia de la RAM son escasos ${ }^{24}$. Usando el software WHONET de acuerdo con el consenso, este estudio presenta las tasas de RAM de los principales agentes etiológicos de las infecciones de las unidades de cuidado intensivo, hospitalización general y servicios de emergencias de los hospitales participantes. La elevada prevalencia de E. coli y $K$. pneumoniae resistentes a cefalosporinas de tercera generación en un rango de 50 a $80 \%$ según el área de localización, la alta resistencia a carbapenémicos (41-72\%) de $P$. aeruginosa, la presencia de $A$. baumannii MDR (>90\%), así como la presencia de $S$. aureus resistente a meticilina (49-79\%) y aislados de E. faecium resistentes a vancomicina (56$76 \%$ ), posicionan a Perú como un país con cifras muy elevadas de microorganismos MDR, semejante a lo que sucede en otros países de Latinoamérica, reforzando la necesidad urgente de implementar un PROA ${ }^{25-27}$. 
De igual manera, se han identificado casos de enterobacterias resistentes a carbapenémicos mediados principalmente por carbapenemasas tipo serina y metalo $\beta$-lactamasas, los que, según lo reportado por Villegas y cols., son un factor de riesgo para la mortalidad de pacientes con infecciones graves ${ }^{28}$, con escasas opciones terapéuticas para su manejo clínico y con gran capacidad de diseminación intra e inter-institucional ${ }^{29}$. Priorizar estrategias costo-efectivas en la prevención y control de infecciones asociadas a la atención en salud, a través de programas multidisciplinarios que involucren todos los actores y recursos involucrados para la atención de los pacientes o su ambiente, es una necesidad para los hospitales en Perú ${ }^{30}$. Producto de la experiencia generada en los diferentes programas de prevención y control de infecciones, los profesionales involucrados en este proceso deben contribuir en el diseño de las estrategias de divulgación, educación y construcción de indicadores de seguimiento al programa, de acuerdo con la experiencia obtenida durante la fase de estructuración de los PROAs ${ }^{31}$.

En el mundo, el incremento en el consumo de antimicrobianos durante los últimos años ha sido dramático; la razón de crecimiento entre los años 2000 y 2015 fue de $65 \%$ pasando de 21,1 a 34,8 millones de DDD consumidas. Este incremento ha sido esencialmente en función de los niveles de consumo de los países de ingresos bajos y medios, superando las tasas de consumo de países de altos ingresos ${ }^{32}$. Medir y monitorizar el consumo de antimicrobianos en hospitales de alta complejidad es reconocido como un componente vital para direccionar las estrategias de los PROAs y determinar su impacto en la epidemiología de la RAM $^{33}$.

Para conocer los patrones de consumo de antimicrobianos según las líneas de acción priorizadas y con la participación del servicio farmacéutico, se realizó el cálculo a través de la metodología de DDD para todos los servicios de hospitalización y cuidados intensivos de pacientes en las instituciones participantes. El consumo promedio de ceftriaxona (13,73 DDD) sumado al uso de carbapenémicos tipo imipenem (4,76 DDD) y meropenem (6,73 DDD), vancomicina (7,35 DDD) y ciprofloxacina (4,2 DDD), permite vigilar y seguir las tendencias de este consumo con el fin de realizar intervenciones de su uso. Estos niveles de consumo pueden superar hasta en $50 \%$ lo reportado en países de la región ${ }^{34}$. La magnitud de la RAM en los hospitales del estudio, la frecuencia de aislamiento de microorganismos MDR y el elevado uso de antimicrobianos de amplio espectro ha llevado a que, durante la fase de fundamentación e implementación temprana, los PROAs prioricen estrategias de control del uso de cefalosporinas de tercera generación y quinolonas, la regulación de carbapenémicos del grupo dos y la pre autorización para el uso de piperacilina/tazobactam, ertapenem o fosfomicina trometamol, de acuerdo a los algoritmos de manejo empírico de las infecciones prevalentes, estratificados por factores de riesgo de los pacientes o niveles de criticidad de los servicios ${ }^{35}$.

La optimización de las dosis, la infusión prolongada y la estandarización de las terapias combinadas frente a sospecha de organismos MDR, también hacen parte de las líneas de acción establecidas para los programas ${ }^{36}$, sumado a la necesidad de incorporar en el formulario institucional otras alternativas terapéuticas para el abordaje de infecciones por microorganismos MDR, tales como polimixina $\mathrm{B}$, fosfomicina IV, ceftazidima/avibactam, ceftolozano/tazobactam y meropenem/vaborbactam una vez que estén disponibles y como parte del manejo de bacterias MDR. Mejorar el acceso a los recursos diagnósticos se consolida también como una necesidad critica para el adecuado funcionamiento de los programas. La auditoría prospectiva de las prescripciones a través del servicio de infectología, ha logrado los primeros resultados durante la fase de implementación temprana de los programas, al promover estrategias de des-escalamiento según evidencia microbiológica, suspensión de doble terapia si se identifica sólo un microrganismo y definición del tiempo de administración del tratamiento de acuerdo a la resolución clínica, así como optimización de la dosis. El hospital C logró disminuir en 30 a 50\% el consumo de antimicrobianos de amplio espectro, mientras que el hospital A logró el impacto en el consumo de imipenem y vancomicina, hallazgos semejantes a los reportados por Reed y cols., en su estudio de evaluación del impacto de combinar las estrategias de restricción con pre-autorización de antimicrobianos en el marco de los PROAs ${ }^{37}$. Si bien el hospital B no logró un impacto significativo temprano, podría lograrlo continuando el PROA por un tiempo mayor; para ellos se requiere hacer una reevaluación de sus recursos ya que este hospital tuvo el menor puntaje ICATB.

En conclusión, en este estudio se presentan los pasos principales para la implementación de un PROA que a través de estrategias diagnósticas de procesos, recursos, líneas de base epidemiológicas, trabajo cooperativo entre equipos multidisciplinarios y priorización de actividades de seguimiento ajustadas a las prácticas de atención de las instituciones, han logrado los primeros resultados en la articulación de los programas, la optimización de las actividades de los servicios de microbiología y farmacia, además de la disminución de los patrones de consumo de antimicrobianos de amplio espectro durante su implementación temprana.

Agradecimientos. A los profesionales adscritos a los servicios de microbiología, farmacia, control de infecciones, inteligencia sanitaria e infectología de los hospitales participantes del estudio. 


\section{Resumen}

Introducción: La resistencia a los antimicrobianos (RAM) es una amenaza para la salud pública mundial Los programas de optimización del uso de antimicrobianos (PROAs) son programas que promueven su adecuado uso, mejoran los resultados clínicos, económicos y contribuyen a contener la RAM. Objetivos: Evaluar las fases de diagnóstico e implementación temprana de los PROAs en tres hospitales de alta complejidad pertenecientes al Sistema de Seguridad Social del Perú. Materiales y Métodos: Estudio multicéntrico, cuasi experimental. La estructuración de los programas, las líneas de base microbiológicas, el consumo de antimicrobianos y los consensos fueron evaluadas durante los períodos de diagnóstico inicial y durante la implementación temprana de los PROAs. Resultados: Con posterioridad a la implementación, los hospitales duplicaron la puntuación de recursos disponibles para los programas (6,75 vs $13,75)$. La prevalencia de enterobacterias portadoras de $\beta$-lactamasas de espectro extendido era de $50-60 \%$, mientras que la resistencia a carbapenémicos en Pseudomonas aeruginosa promedió el $69 \%$. La dosis diaria definida de ceftriaxona fue de 13,63 , de 7,35 para vancomicina y 6,73 para meropenem en promedio. Los hospitales A y $\mathrm{C}$ disminuyeron el uso de antimicrobianos en 30 a $50 \%$. Discusión: A través de estrategias diseñadas por equipos multidisciplinarios para implementar los PROAs, se logró disminuir tempranamente el consumo de antimicrobianos de amplio espectro.

\section{Referencias bibliográficas}

1.- Allcock S, Young E H, Holmes M, Gurdasani D, Dougan G, Sandhu M S, et al. Antimicrobial resistance in human populations: challenges and opportunities. Glob Health Epidemiol Genomics 2017; 2: e4. doi: https://doi. org/10.1017/gheg.2017.4.

2.- Tamma P D, Cosgrove S E. Antimicrobial stewardship. Infect Dis Clin North Am 2011; 25 (1): 245-60. doi: 10.1016/j.idc.2010.11.011.

3.- $\mathrm{WHO} \mid$ Antimicrobial resistance: global report on surveillance 2014 [Internet]. WHO. [cited 2018 Jul 16]. Available from: http:// www.who.int/drugresistance/documents/ surveillancereport/en/

4.- Revie N M, Iyer K R, Robbins N, Cowen L E. Antifungal drug resistance: evolution, mechanisms and impact. Curr Opin Microbiol. 2018; 45: 70-6. doi: 10.1016/j.mib.2018.02.005.

5.- $\quad$ WHO $\mid$ Strategic and Technical Advisory Group (STAG) on antimicrobial resistance [Internet]. WHO. [citado el 16 de julio de 2018]. Available from: http://www.who.int/antimicrobialresistance/events/stag/en/

6.- Birgand G, Castro-Sánchez E, Hansen S, Gastmeier P, Lucet J-C, Ferlie E, et al. Comparison of governance approaches for the control of antimicrobial resistance: Analysis of three European countries. Antimicrob Resist Infect Control [Internet]. 2018 Feb 20 [citado el 17 de abril de 2018];7. Available from: https://www.ncbi.nlm.nih.gov/pmc/articles/ PMC5819189/

7.- Home |AMR Review [Internet]. [cited 2018 Jul 16]. Available from: https://amr-review.org/

8.- Bloom G, Merrett G B, Wilkinson A, Lin V, Paulin S. Antimicrobial resistance and universal health coverage. BMJ Glob Health. 2017; 2:e000518. doi:10.1136/bmjgh-2017-000518

9.- Karam G, Chastre J, Wilcox M H, Vincent J-L.
Antibiotic strategies in the era of multidrug resistance. Crit Care 2016; (20): 1: 136. doi: 10.1186/s13054-016-1320-7.

10.- Founou R C, Founou L L, Essack S Y. Clinical and economic impact of antibiotic resistance in developing countries: A systematic review and meta-analysis. PLoS ONE [Internet]. 2017; 12 (12): e0189621. doi: 10.1371/journal. pone.0189621.

11.- Berkelman R, Cassell G, Specter S, Hamburg M, Klugman K. The "Achilles heel" of global efforts to combat infectious diseases. Clin Infect Dis 2006; 42 (10 ): 1503-4. doi: 10.1086/504494.

12.- Bertollo L G, Lutkemeyer D S, Levin A S. Are antimicrobial stewardship programs effective strategies for preventing antibiotic resistance? A systematic review. Am J Infect Control. 2018; 46 (7): 824-36. doi: 10.1016/j.ajic.2018.01.002.

13.- Özgenç O. Methodology in improving antibiotic implementation policies. World J Methodol. 2016; 6(2): 143-53. doi: 10.5662/ wjm.v6.i2.143.

14.- Filice G, Drekonja D, Greer N, Butler M, Wagner B, MacDonald R, et al. Antimicrobial Stewardship Programs in Inpatient Settings: A Systematic Review [Internet]. Washington (DC): Department of Veterans Affairs (US); 2013 [citado el 18 de abril de 2018]. (VA Evidence-based Synthesis Program Reports). Available from: http://www.ncbi.nlm.nih.gov/ books/NBK253513/

15.- Étienne P, Roger P-M, Brofferio P, Labate $\mathrm{C}$, Blanc V, Tiger F, et al. Antimicrobial stewardship program and quality of antibiotic prescriptions. Médecine Mal Infect 2011; 41 (11): 608-12. doi: 10.1016/j. medmal.2011.07.010.

16.- García Calvente M M, Mateo Rodríguez I. El grupo focal como técnica de investigación cualitativa en salud: diseño y puesta en práctica.
Aten Primaria 2000; 25 (3): 181-6. http://www. unidaddocentemfyclaspalmas.org.es/resources/ 5+Aten+Primaria $+2000 .+$ Grupo + Focal + Dise $\$$ $\mathrm{C} 3 \$ \mathrm{~B} 1 \mathrm{o}+\mathrm{y}+$ Practica.pdf

17.- OMS | Vigilancia del uso de los antimicrobianos [Internet]. WHO. [citado el 18 de abril de 2018]. Available from: http://www. who.int/drugresistance/surveillance_use/es/

18.- Dik J-WH, Hendrix R, Poelman R, Niesters H G, Postma M J, Sinha B, et al. Measuring the impact of antimicrobial stewardship programs. Expert Rev Anti Infect Ther. 2016; 14(6): 56975. doi: 10.1080/14787210.2016.1178064.

19.- Core Elements of Hospital Antibiotic Stewardship Programs | Antibiotic Use | CDC [Internet]. 2017 [citado el 23 de abril de 2018]. Available from: https://www.cdc.gov/antibioticuse/healthcare/implementation/core-elements. $\mathrm{html}$

20.- Leung V, Wu JH-C, Langford B J, Garber G. Landscape of antimicrobial stewardship programs in Ontario: a survey of hospitals. CMAJ Open 2018; 6 (1): E71-6. doi: 10.9778/ cmajo.20170111

21.- Bal A M, Gould I M. Antibiotic stewardship: overcoming implementation barriers. Curr Opin Infect Dis 2011; 24 (4): 357-62. doi: 10.1097/ QCO.0b013e3283483262.

22.- Kim J, Craft D W, Katzman M. Building an Antimicrobial Stewardship Program: Cooperative roles for pharmacists, infectious diseases specialists, and clinical microbiologists. Lab Med 2015; 46(3): e65-71. doi: 10.1309/LMCOSHRJBY0ONHI9.

23.- Johnson A P, Muller-Pebody B, Budd E, Ashiru-Oredope D, Ladenheim D, Hain D, et al. Improving feedback of surveillance data on antimicrobial consumption, resistance and stewardship in England: putting the data at your Fingertips. J Antimicrob Chemother 2017; 72 (4): 953-6. doi: 10.1093/jac/dkw536. 
24.- Cox J A, Vlieghe E, Mendelson M, Wertheim H, Ndegwa L, Villegas M V, et al. Antibiotic stewardship in low- and middle-income countries: the same but different? Clin Microbiol Infect. 2017; 23 (11): 812-8. doi: 10.1016/j.cmi.2017.07.010.

25.- Karlowsky J A, Hoban D J, Hackel M A, Lob S H, Sahm D F. Resistance among Gramnegative ESKAPE pathogens isolated from hospitalized patients with intra-abdominal and urinary tract infections in Latin American countries: SMART 2013-2015. Braz J Infect Dis. 2017; 21 (3): 343-8. doi: 10.1016/j. bjid.2017.03.006.

26.- Seas C, García C, Salles MJ, Labarca J, Luna C, Alvarez-Moreno C, et al. Staphylococcus aureus bloodstream infections in Latin America: results of a multinational prospective cohort study. J Antimicrob Chemother. 2018; 73 (1): 212-22. doi: 10.1093/jac/dkx350.

27.- Pfaller M A, Huband M D, Streit J, Flamm R K, Sader H S. Surveillance of tigecycline activity tested against clinical isolates from a global (North America, Europe, Latin America and Asia-Pacific) collection (2016). Int J Antimicrob Agents. 2018; 51(6): 848-53. doi: 10.1016/j.ijantimicag.2018.01.006.
28.- Villegas M V, Pallares C J, EscandónVargas K, Hernández-Gómez C, Correa A, Álvarez C, et al. Characterization and clinical impact of bloodstream infection caused by carbapenemase-producing Enterobacteriaceae in seven Latin American countries. PLOS ONE 2016; 11( 4): e0154092. doi: 10.1371/journal. pone. 0154092

29.- Rojas L J, Weinstock G M, De La Cadena E, Díaz L, Ríos R, Hanson B M, et al. An analysis of the epidemic of Klebsiella pneumoniae carbapenemase-producing $K$. pneumoniae: Convergence of two evolutionary mechanisms creates the "Perfect Storm." J Infect Dis 2017; 217(1): 82-92. doi: 10.1093/infdis/jix524.

30.- Sydnor E R M, Perl T M. Hospital epidemiology and infection control in acutecare settings. Clin Microbiol Rev 2011; 24 (1): 141-73. doi: 10.1128/CMR.00027-10.

31.- Nagel J L, Kaye K S, LaPlante K L, Pogue J M. Antimicrobial stewardship for the infection control practitioner. Infect Dis Clin North Am 2016; 30 (3): 771-84. doi: 10.1016/j. idc.2016.04.012.

32.- Klein E Y, Van Boeckel T P, Martínez E M, Pant S, Gandra S, Levin S A, et al. Global increase and geographic convergence in antibiotic consumption between 2000 and 2015. Proc Natl Acad Sci USA 2018; 115 (15): E3463-70. doi: 10.1073/pnas.1717295115.

33.- Ibrahim O M, Polk R E. Benchmarking antimicrobial drug use in hospitals. Expert Rev Anti Infect Ther 2012; 10 (4): 445-57. doi: 10.1586/eri.12.18.

34.- Domínguez I, Rosales R, Cabello Á, Bavestrello L, Labarca J. Evaluation of antimicrobial consumption en 15 Chilean hospitals: Results of a collaborative work, 2013. Rev Chilena Infectol 2016; 33 (3): $307-$ 12. doi: $10.4067 / \mathrm{S} 0716-10182016000300010$.

35.- Bogan C, Marchaim D. The role of antimicrobial stewardship in curbing carbapenem resistance. Future Microbiol 2013; 8 (8): 979-91. doi: 10.2217/fmb.13.73.

36.- Vitrat V, Hautefeuille S, Janssen C, Bougon D, Sirodot M, Pagani L. Optimizing antimicrobial therapy in critically ill patients. Infect Drug Resist. 2014; 7: 261-71. doi: 10.2147/IDR. S44357.

37.- Reed E E, Stevenson K B, West J E, Bauer K A, Goff D A. Impact of formulary restriction with prior authorization by an antimicrobial stewardship program. Virulence. 2013; 4 (2): 158-62. doi: 10.4161 /viru.21657. 